\title{
Tip-Enhanced Raman Nanographs of Plasmonic Silver Nanoparticles
}

\author{
Ashish Bhattarai, ${ }^{1}$ Irina V. Novikova, ${ }^{2}$ and Patrick Z. El-Khoury ${ }^{1, *}$
}

${ }^{1}$ Physical Sciences Division and ${ }^{2}$ Environmental Molecular Sciences Laboratory, Pacific

Northwest National Laboratory, P.O. Box 999, Richland, WA 99352, USA

*patrick.elkhoury@pnnl.gov 

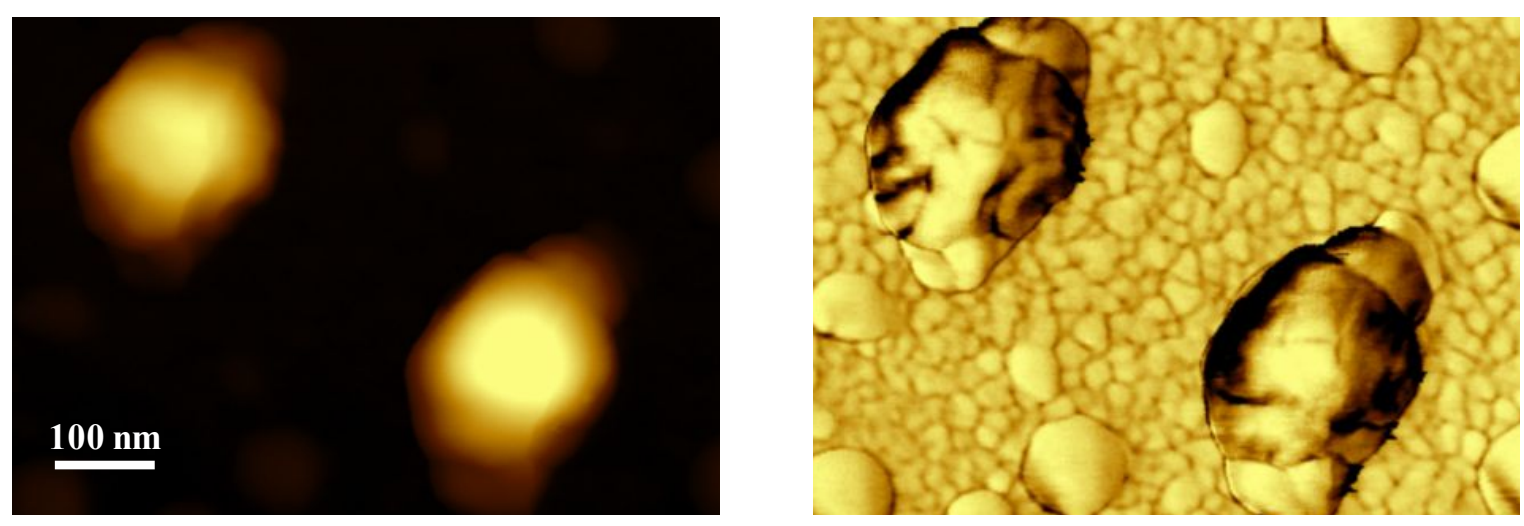

Figure S1: AFM height (left panel) and phase (right panel) images of the particles analyzed in Figure 2 of the main text. 

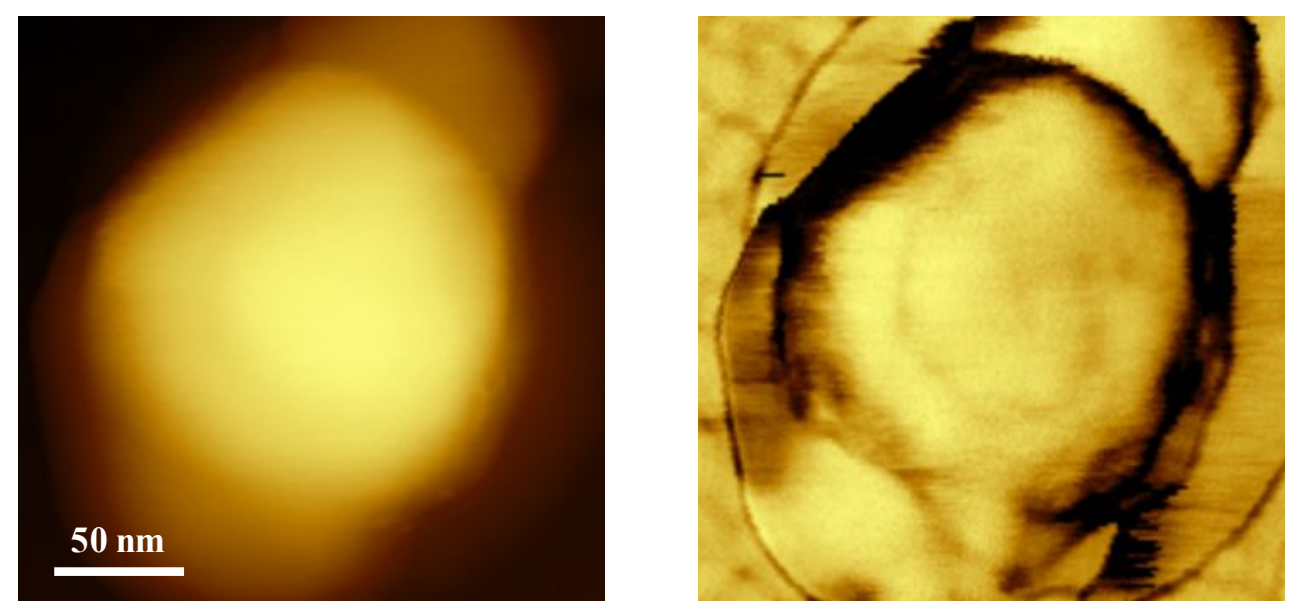

Figure S2: AFM height (left panel) and phase (right panel) images of the particles analyzed in Figure 4 of the main text. 

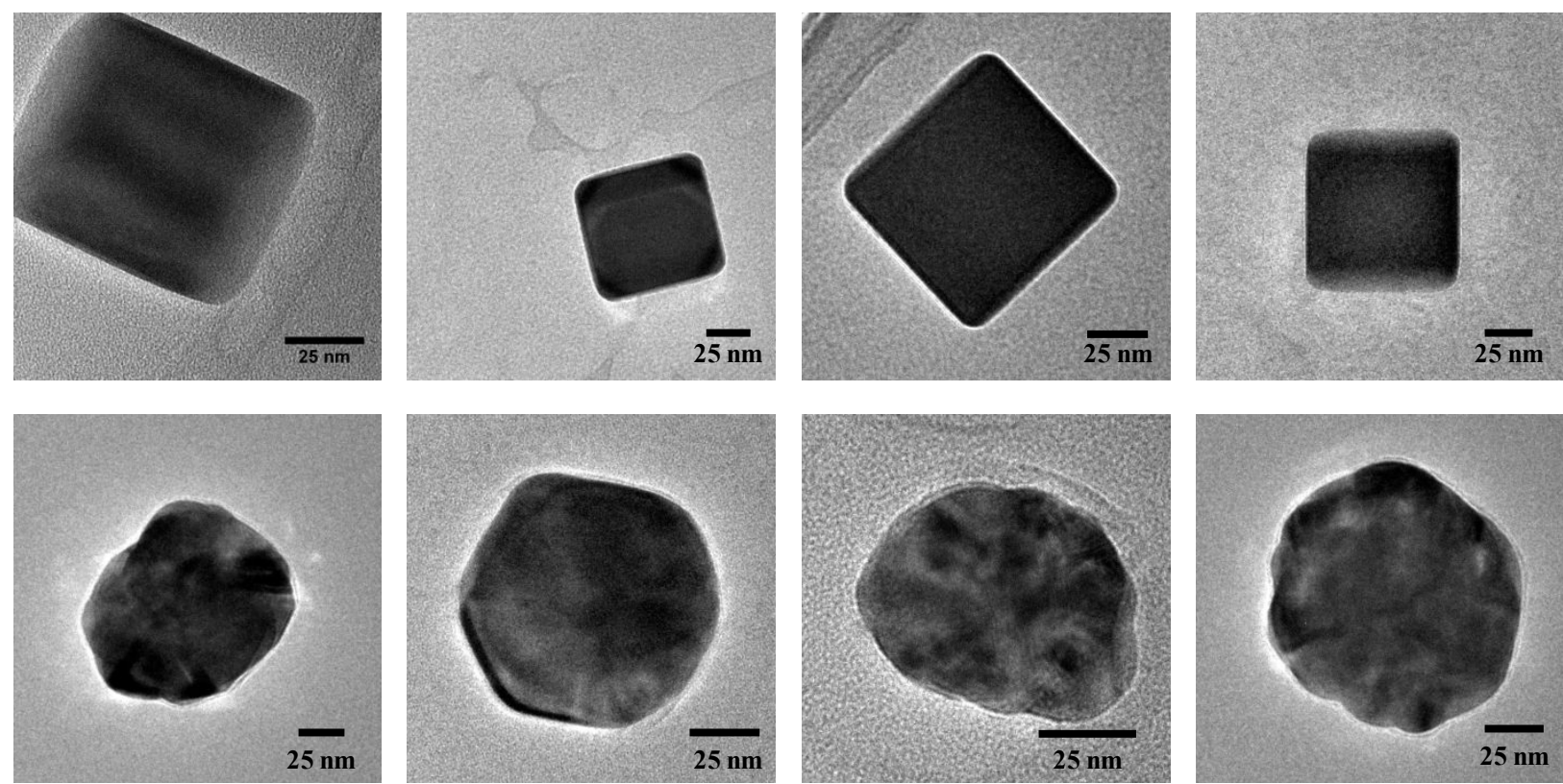

Figure S3: Representative TEM images of the silver nanocubes (top row) and nanoparticles (bottom row) that were used in this study. 

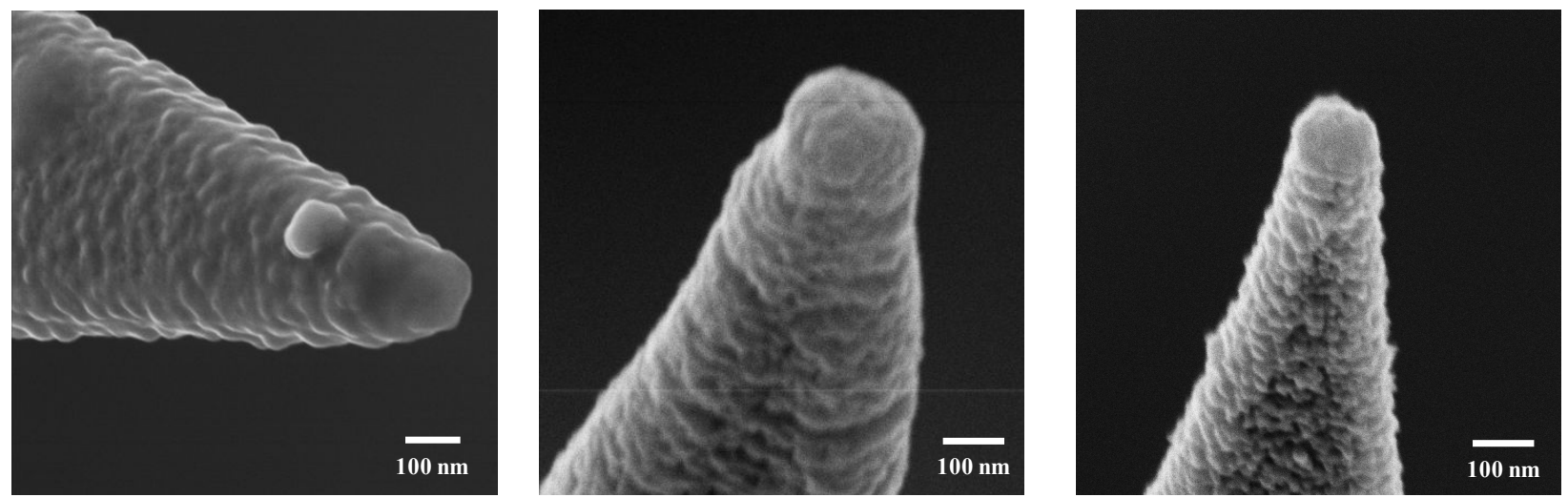

Figure S4: Representative helium ion images of the Au-coated AFM probes that were used in this study. 